Old Dominion University

ODU Digital Commons

\title{
Muon Capture in Nuclei: An ab initio Approach Based on Green's Function Monte Carlo Methods
}

\author{
A. Lovato \\ N. Rocco \\ Rocco Schiavilla \\ Old Dominion University, rschiavi@odu.edu
}

Follow this and additional works at: https://digitalcommons.odu.edu/physics_fac_pubs

Part of the Nuclear Commons

\section{Original Publication Citation}

Lovato, A., Rocco, N., \& Schiavilla, R. (2019). Muon capture in nuclei: An ab initio approach based on green's function Monte Carlo methods. Physical Review C, 100(3), 035502. doi:10.1103/

PhysRevC.100.035502

This Article is brought to you for free and open access by the Physics at ODU Digital Commons. It has been accepted for inclusion in Physics Faculty Publications by an authorized administrator of ODU Digital Commons. For more information, please contact digitalcommons@odu.edu. 


\title{
Muon capture in nuclei: An ab initio approach based on Green's function Monte Carlo methods
}

\author{
A. Lovato $\odot,{ }^{1,2}$ N. Rocco, ${ }^{2,3}$ and R. Schiavilla ${ }^{4,5}$ \\ ${ }^{1}$ INFN-TIFPA Trento Institute of Fundamental Physics and Applications, 38123 Trento, Italy \\ ${ }^{2}$ Physics Division, Argonne National Laboratory, Argonne, Illinois 60439, USA \\ ${ }^{3}$ Theoretical Physics Department, Fermi National Accelerator Laboratory, Batavia, Illinois 60510, USA \\ ${ }^{4}$ Department of Physics, Old Dominion University, Norfolk, Virginia 23529, USA \\ ${ }^{5}$ Theory Center, Jefferson Lab, Newport News, Virginia 23606, USA
}

(Received 27 March 2019; revised manuscript received 24 June 2019; published 12 September 2019)

\begin{abstract}
An $a b$ initio Green's function Monte Carlo (GFMC) method is introduced for calculating total rates of muon weak capture in light nuclei with mass number $A \leqslant 12$. As a first application of the method, we perform a calculation of the rate in ${ }^{3} \mathrm{H}$ and ${ }^{4} \mathrm{He}$ in a dynamical framework based on realistic two- and three-nucleon interactions and realistic nuclear charge-changing weak currents. The currents include one- and two-body terms induced by $\pi$ - and $\rho$-meson exchange, and $N$-to- $\Delta$ excitation, and are constrained to reproduce the empirical value of the Gamow-Teller matrix element in tritium. We investigate the sensitivity of theoretical predictions to current parametrizations of the nucleon axial and induced pseudoscalar form factors as well as to two-body contributions in the weak currents. The large uncertainties in the measured ${ }^{4} \mathrm{He}$ rates obtained from bubble-chamber experiments (carried out over 50 years ago) prevent us from drawing any definite conclusions. No data exist for ${ }^{3} \mathrm{H}$, but results are compared to those of a recent Faddeev calculation as a validation of the present GFMC method.
\end{abstract}

DOI: 10.1103/PhysRevC.100.035502

\section{INTRODUCTION}

Negative muons passing through matter can be captured into high-lying atomic orbitals, from where they rapidly cascade down into the $1 s$ orbital. There they either decay via the process $\mu^{-} \rightarrow e^{-} \bar{v}_{e} v_{\mu}$ with a rate which is almost the same as in free space [1] or are captured by the nucleus in a weak-interaction process, resulting in the change of one of the protons into a neutron at a rate that is proportional to $Z^{4}$ [2], where $Z$ is the nucleus' proton number and which, at least for light nuclei, is much smaller than the free decay rate.

In the nuclear capture, the muon rest mass $\left(m_{\mu}\right)$ is converted in energy shared by the emitted (muon) neutrino and recoiling final nucleus. Since $m_{\mu} \approx 105 \mathrm{MeV}$, a calculation of the total inclusive rate-i.e., summed over all final states-requires, in principle, knowledge of both the low-lying discrete states and higher-energy continuum spectrum of the final nucleus. In $a b$ initio dynamical approaches based on realistic nuclear interactions, the solution of the scattering problem poses a significant challenge, even for capture in nuclei as light as ${ }^{3} \mathrm{He}$ and ${ }^{3} \mathrm{H}$. Indeed, while accurate theoretical estimates of the ${ }^{3} \mathrm{He}\left(\mu^{-}, v_{\mu}\right)^{3} \mathrm{H}$ rate (a transition only involving bound states) have been made since the early 1990s [3-6], it is only recently that studies based on the Faddeev method and accounting for the contributions to the rate from the breakup channels of ${ }^{3} \mathrm{He}$ (into ${ }^{2} \mathrm{H}+n$ and ${ }^{1} \mathrm{H}+2 n$ ) and ${ }^{3} \mathrm{H}$ (into $3 n$ ) have appeared in the literature, respectively in Refs. [7] and [8].

The other important aspect of muon capture has to do with the description of the nuclear charge-changing weak current responsible for the $p-n$ conversion. Its dominant one-body term is associated with the matrix element $\left\langle n\left|\bar{d} \gamma^{\mu}\left(1-\gamma_{5}\right)\right| p\right\rangle$ and is parametrized in terms of four form factors (FFs). Two of these, $F_{1}\left(q^{2}\right)$ and $F_{2}\left(q^{2}\right)\left(q^{2}\right.$ is the lepton four-momentum transfer), enter the vector component and are related to the isovector electromagnetic FFs by the conserved-vectorcurrent (CVC) constraint. The remaining two, the axial and induced pseudoscalar FFs, respectively $G_{A}\left(q^{2}\right)$ and $G_{P}\left(q^{2}\right)$, characterize the axial component. The $F_{1}\left(q^{2}\right)$ and $F_{2}\left(q^{2}\right)$ FFs are well known over a broad range of momentum transfers from elastic electron scattering off protons and deuterons [9]. The value $g_{A}$ of the axial $\mathrm{FF}$ at vanishing $q^{2}$ is precisely determined from neutron $\beta$ decay, $g_{A}=1.2723(23)$ [10], while the $q^{2}$ dependence is parametrized by a dipole form with a cutoff $\Lambda_{A} \approx 1 \mathrm{GeV}$ as obtained in analyses of pion electroproduction data [11] and direct measurements of $v_{\mu} / \bar{v}_{\mu}-p$ [12] and quasielastic $v_{\mu}-d$ [13-15] scattering cross sections. A recent measurement of muon capture in hydrogen by the MuCap collaboration at Paul Scherrer Institute [16] has led to a precise determination of the $G_{P}\left(q^{2}\right) \mathrm{FF}$ (the least well experimentally known of the four) albeit at the single kinematical point $q_{0}^{2}=-0.88 m_{\mu}^{2}: G_{P}\left(q_{0}^{2}\right)=8.06 \pm 0.55$, a value that is consistent with theoretical predictions derived from chiral perturbation theory [17-19].

In the nuclear charge-changing weak current, in addition to one-body, there are two-body terms that arise quite naturally in the conventional meson-exchange picture; for reviews see Refs. [20,21], as well as in more modern approaches based on chiral effective field theory [22-29]. Those in the vector sector are related by CVC to the isovector two-body electromagnetic currents, notably the long-range currents induced 
by pion exchange. By now, there is a substantial body of experimental evidence for their presence from a variety of photo- and electronuclear transitions in nuclei, including, among others, thermal neutron radiative captures on hydrogen and helium isotopes, magnetic moments, and $M 1$ transition rates in light nuclei, elastic, and transition magnetic form factors of few-nucleon systems, and, last, transverse response functions measured in quasielastic $\left(e, e^{\prime}\right)$ scattering off light nuclei (see Refs. [30-32] for reviews which include extensive listings of original references). In the axial sector, however, this evidence is not as well established, in that discrepancies between experimental data and theoretical predictions obtained with one-body currents are not as large as in the electromagnetic case and concern, primarily, the very low momentum and energy transfers of relevance in $\beta$ decays of very light nuclei [33-35] and, recently, some heavier nuclei as well [36]. In particular, it is worthwhile noting here that the relative sign between the one- and two-body axial corrections, at least in the mass range $3<A \leqslant 10$, is calculated to be the same in Ref. [35] but generally opposite in Ref. [36] (the tritium and ${ }^{8} \mathrm{He}$ Gamow-Teller matrix elements, the former of which is actually fitted, are the only exceptions). The origin of this difference is yet to be clarified. Carrying out benchmark calculations with different interactions, currents, and manybody methods would be very helpful. We should also point out that results from earlier studies [37-39] of muon-capture rates in ${ }^{12} \mathrm{C},{ }^{16} \mathrm{O}$, and ${ }^{40} \mathrm{Ca}$, based on the continuum random-phase and quasi-particle random-phase approximations, reproduce the experimental data without requiring a quenching of $g_{A}$.

Given the above context, the objectives of the present work are twofold: (i) to formulate a Green's function Monte Carlo (GFMC) method for calculating, $a b$ initio, inclusive muon-capture rates in nuclei in the mass range $A=3-12$ and (ii) to test our present modeling of the nuclear chargechanging weak current by comparing theoretical results with available experimental data. This will validate the modeling in a range of momentum and energy transfers that is intermediate between those relevant, at the low end, in $\beta$ decays and, at the high end, in neutrino scattering. We begin by establishing the kinematics of the process and expressing the rate in a form amenable to a GFMC calculation, and then focus on muon capture in ${ }^{3} \mathrm{H}$ and ${ }^{4} \mathrm{He}$ as first practical applications of the method.

\section{METHODOLOGY}

The muon is captured by the nucleus from an atomic orbital, and its momentum and energy are denoted by $\mathbf{k}_{\mu}$ and $E_{\mu}$, with the understanding that $k_{\mu} \rightarrow 0$, since the muon orbital velocity is of order $Z \alpha \ll 1$ for light atoms. The muon-neutrino momentum and energy are denoted as $\mathbf{k}_{v}$ and $E_{v}\left(E_{v}=k_{v}\right)$, and the masses of the proton and neutron as $m_{p}$ and $m_{n}$. In the capture process a proton in the initial atom is converted into a neutron, and energy conservation requires

$$
\Delta m+E_{i}=E_{v}+E_{f}, \quad \Delta m=m_{\mu}+m_{p}-m_{n},
$$

where $E_{i}$ is the internal energy (of electrons and nucleons) of the initial atom, and $E_{f}$ is the energy of the final atom including both its internal and recoil energies. Of course, binding energies of electrons, at least for light atoms, are of the order of tens of eV's, and therefore negligible when compared to those of nucleons.

The transition amplitude for capture at leading order is given by

$$
T_{f i}=\frac{G_{V}}{\sqrt{2}} \psi(0)\left[\bar{u}_{\mathbf{k}_{v} h_{v}} \gamma_{\sigma}\left(1-\gamma_{5}\right) u_{\mathbf{k}_{\mu} s_{\mu}}\right] j_{f i}^{\sigma}\left(-\mathbf{k}_{v}\right),
$$

where $G_{V}$ is the vector coupling constant for semileptonic weak interactions $\left(G_{V} \approx 1.1363 \times 10^{-5} \mathrm{GeV}^{-2}[40]\right), u_{\mathbf{k}_{\mu} s_{\mu}}$ and $u_{\mathbf{k}_{v} h_{v}}$ are the spinors (normalized here as $u^{\dagger} u=1$ ) of, respectively, the muon with spin projection $s_{\mu}$ and neutrino with helicity $h_{v}$, and $j_{f i}^{\sigma}$ is the matrix element of the hadronic charge-lowering weak current,

$$
j_{f i}^{\sigma}\left(-\mathbf{k}_{v}\right)=\left\langle-\mathbf{k}_{v}, f\left|\int d \mathbf{x} e^{-i \mathbf{k}_{v} \cdot \mathbf{x}} j^{\sigma}(\mathbf{x})\right| i, J_{i} M_{i}\right\rangle .
$$

Since the matrix element $\left\langle f\left|j^{\sigma}(\mathbf{x})\right| i\right\rangle$ is localized over length scales of a few fm's, the atomic wave function $\psi(x)$ of the muon has been approximated by its value at the origin, $\psi(0)=(Z \alpha \mu)^{3} / \pi$, where $\alpha$ is the fine structure constant and $\mu$ is the reduced mass of the muon relative to the initial nucleus with $Z$ protons. Note that the two-component spinstate $\chi_{s_{\mu}}$ of the muon has been replaced by the spinor, which is justified in the limit $k_{\mu} \rightarrow 0$ (and also helpful for carrying out the sums over spins by standard trace techniques). Finally, $\left|i, J_{i} M_{i}\right\rangle$ and $\left|-\mathbf{k}_{v}, f\right\rangle$ are, respectively, the initial nuclear state with spin and spin-projection $J_{i} M_{i}$ and the final nuclear state recoiling with momentum $-\mathbf{k}_{v}$ with quantum numbers collectively specified by the label $f$.

The transition rate, when averaged over the spin projections of the initial nucleus and muon, and summed over those of the final nucleus, is independent of the $\hat{\mathbf{k}}_{v}$ direction and reduces to the well-known expression in terms of Coulomb, longitudinal, electric, and magnetic multipoles (see, for example, Ref. [41]). In the present context, however, we find it convenient to express this rate (differential in the emitted neutrino energy, but integrated over the solid angle) in terms of five response functions,

$$
\begin{aligned}
\frac{d \Gamma}{d E_{v}}= & \frac{G_{V}^{2}}{2 \pi}|\psi(0)|^{2} E_{v}^{2}\left[R_{00}\left(E_{v}\right)+R_{z z}\left(E_{v}\right)+R_{0 z}\left(E_{v}\right)\right. \\
& \left.+R_{x x}\left(E_{v}\right)-R_{x y}\left(E_{v}\right)\right],
\end{aligned}
$$

with

$$
\begin{aligned}
& R_{00}\left(E_{v}\right)=\overline{\sum_{i, f}} \delta(\cdots)\left|j_{f i}^{0}\left(-\mathbf{k}_{v}\right)\right|^{2}, \\
& R_{z z}\left(E_{v}\right)=\overline{\sum_{i, f}} \delta(\cdots)\left|j_{f i}^{\|}\left(-\mathbf{k}_{v}\right)\right|^{2} \\
& R_{0 z}\left(E_{v}\right)=-\overline{\sum_{i, f}} \delta(\cdots) 2 \operatorname{Re}\left[j_{f i}^{0}\left(-\mathbf{k}_{v}\right) j_{f i}^{\| *}\left(-\mathbf{k}_{v}\right)\right], \\
& R_{x x}\left(E_{v}\right)=\overline{\sum_{i, f}} \delta(\cdots)\left|\mathbf{j}_{f i}^{\perp}\left(-\mathbf{k}_{v}\right)\right|^{2}, \\
& R_{x y}\left(E_{v}\right)=i \overline{\sum_{i, f}} \delta(\cdots) \hat{\mathbf{k}}_{v} \cdot\left[\mathbf{j}_{f i}^{\perp}\left(-\mathbf{k}_{v}\right) \times \mathbf{j}_{f i}^{\perp *}\left(-\mathbf{k}_{v}\right)\right],
\end{aligned}
$$


TABLE I. The inclusive muon rates in ${ }^{4} \mathrm{He}$ obtained by including one-body (1b) only and both one- and two-body ( $\left.2 \mathrm{~b}\right)$ terms in the vector (V) and axial (A) components of the charge-changing (CC) weak current. The $1 \mathrm{~b}$ and $2 \mathrm{~b}$ rates obtained with the full CC current and the $\widetilde{\mathrm{CC}}$ current without the induced pseudoscalar term are compared to available experimental values and older theoretical estimates.

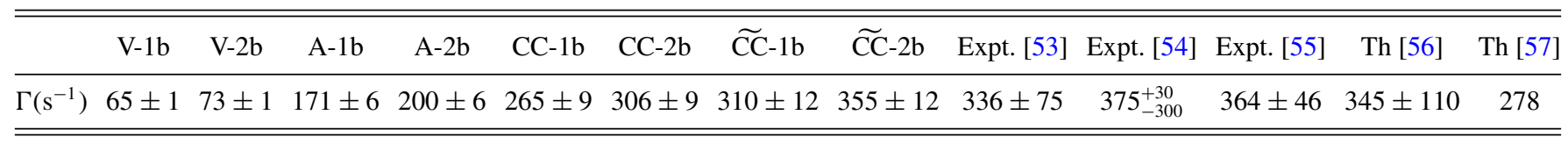

where we have introduced the unit vector $\hat{\mathbf{k}}_{v}=\mathbf{k}_{v} / E_{v}$, the longitudinal and transverse components of the current, respectively $j_{f i}^{\|}=\hat{\mathbf{k}}_{v} \cdot \mathbf{j}_{f i}$ and $\mathbf{j}_{f i}^{\perp}=\mathbf{j}_{f i}-\hat{\mathbf{k}}_{v} j_{f i}^{\|}$, and the short-hand notation $\delta(\cdots)$ for the energy-conserving $\delta$ function resulting from Eq. (1). The bar over the summation symbol implies the average over (nuclear) spin projections indicated earlier.

As they stand, a calculation of these response functions by GFMC methods [32,42-45] is not possible, since the lepton momentum and energy transfers, respectively $\mathbf{q}$ and $\omega$, in the weak capture (like in a photoabsorption process) are not independent variables; indeed, $\mathbf{q}=-E_{v} \hat{\mathbf{k}}_{v}$ and $\omega=m_{\mu}-E_{v}$. To circumvent this difficulty, we consider instead (in a schematic notation)

$$
R_{\alpha \beta}(q, \bar{\omega})=\overline{\sum_{i f}} \delta\left(\bar{\omega}+E_{i}-E_{f}\right) O_{f i}^{\alpha}(\mathbf{q}) O_{f i}^{\beta *}(\mathbf{q}),
$$

with $\mathbf{q}=-E_{v} \hat{\mathbf{k}}_{v}$ and $\bar{\omega}$ taken as independent variables. We carry out the Laplace transform

$$
\begin{aligned}
& E_{\alpha \beta}(q, \tau)=\int_{0}^{\infty} d \bar{\omega} e^{-\tau \bar{\omega}} R_{\alpha \beta}(q, \bar{\omega}) \\
& =\overline{\sum_{i}}\left\langle i\left|O^{\beta \dagger}(\mathbf{q}) e^{-\tau\left(H-E_{i}\right)} O^{\alpha}(\mathbf{q})\right| i\right\rangle,
\end{aligned}
$$

by evaluating the expectation value in the second line above with stochastic techniques [46], invert the resulting Euclidean response function $E_{\alpha \beta}(q, \tau)$ by maximum-entropy methods $[43,47,48]$ to obtain back $R_{\alpha \beta}(q, \bar{\omega})$, and, finally, interpolate the latter at $\bar{\omega}=\omega+m_{p}-m_{n}=\Delta m-E_{v}$ to determine the response $R_{\alpha \beta}\left(E_{v}\right)$ of interest here. No approximations are made beyond those inherent to the modeling of the nuclear Hamiltonian and weak current; in particular, interaction effects in the discrete and continuum spectrum of the final nuclear system are fully and exactly accounted for.

The dynamical framework adopted in the present work is based on a realistic Hamiltonian including the Argonne $v_{18}$ two-nucleon [49] (AV18) and Illinois-7 three-nucleon [50] (IL7) interactions and on realistic charge-changing weak currents with one- and two-body terms, see Ref. [51] for a recent overview and a listing of explicit expressions. The (vector and axial) one-body terms $j_{1 \mathrm{~b}}^{\sigma}$ follow from a nonrelativistic expansion of the single-nucleon (charge-changing) weak current, in which corrections proportional up to the inverse-square of the nucleon mass are retained. The twobody currents $j_{2 \mathrm{~b}}^{\sigma}$ consist of contributions associated with (effective) $\pi$ - and $\rho$-meson exchanges and $N$-to- $\Delta$ excitation terms, treated in the static limit. In the axial component, a $\rho \pi$ transition mechanism is also included. Configurationspace representations of these currents (used in the actual calculations below) are regularized by a prescription which, albeit model dependent, is nevertheless designed to make, by construction, their short-range behavior consistent with that of the two-nucleon interaction-the AV18. In the $N$-to- $\Delta$ axial current, the value for the transition (axial) coupling constant is determined by reproducing the measured Gamow-Teller matrix element contributing to tritium $\beta$ decay [51] (within the present dynamical framework). The level of quantitative success these currents have achieved, when used in combination with the AV18+IL7 Hamiltonian, in accurately predicting many electroweak properties of $s$ - and $p$-shell nuclei up to ${ }^{12} \mathrm{C}$ is illustrated in Refs. [30,32] and references therein.

\section{RESULTS AND CONCLUSIONS}

Having set up the formalism and specified the dynamical framework, we now proceed to discuss an application of the method to muon capture in ${ }^{3} \mathrm{H}$ and ${ }^{4} \mathrm{He}$. There are no experimental data for ${ }^{3} \mathrm{H}$, and the main reason for calculating its rate is to benchmark the present method against the Faddeev approach used in Ref. [8]. In the case of ${ }^{4} \mathrm{He}$, as noted by Measday in his review [52], the only available measurements of the total rate are from experiments in the 1960s with helium bubble chambers and helium gas scintillating targets [53-55] and have large errors, see Table I. The only theoretical estimates we are aware of are from Caine and Jones [56] and Walecka [57]; the former based on closure approximations is rather uncertain, while the latter obtained with the Foldy-Walecka sum rules for the giant dipole excitation turns out to be remarkably close to the value we calculate almost 50 years later!

The calculation of the ${ }^{3} \mathrm{H}$ and ${ }^{4} \mathrm{He}$ Euclidean responses in Eq. (11) is carried out with GFMC methods [42-45] similar to those used in projecting out the exact ground state of a Hamiltonian from a trial state [58]. It proceeds in two steps. First, an unconstrained imaginary-time propagation of the initial bound state state $|i\rangle$, represented here by accurate ${ }^{3} \mathrm{H}$ and ${ }^{4} \mathrm{He}$ variational Monte Carlo wave function (rather than their exact GFMC counterparts), is performed and saved. Next, the states $O^{\alpha}(\mathbf{q})|i\rangle$ are evolved in imaginary time following the path previously saved. During this latter imaginary-time evolution, scalar products of $\exp \left[-\left(H-E_{i}\right) \tau_{i}\right] O^{\alpha}(\mathbf{q})|i\rangle$ with $O^{\beta}\left(\mathbf{q}|i\rangle\right.$ are evaluated on a grid of $\tau_{i}$ values, and from these scalar products estimates for $E_{\alpha \beta}\left(q, \tau_{i}\right)$ are obtained. Since nuclear charge is not conserved in the process, the need arises to propagate intermediate states with a different number of protons and neutrons relative to the ground state-how this is implemented in the present calculations is discussed briefly in the Supplemental Material [59]. The statistical errors associated with the GFMC evolution remain modest, even at values of $\tau$ as large as $0.1 \mathrm{MeV}^{-1}$, the endpoint of the $\tau$ grid. Maximum entropy methods are employed "to invert" $E_{\alpha \beta}(q, \tau)$ and obtain the corresponding $R_{\alpha \beta}(q, \bar{\omega})$ [43]. 


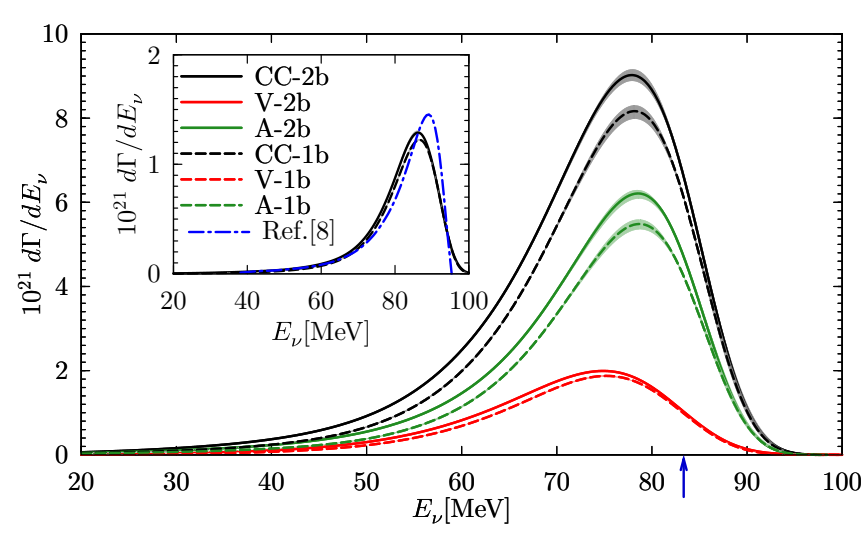

FIG. 1. The differential rates obtained with one-body (1b) only and both one- and two-body (2b) terms in the vector (V) and axial (A) components of the charge-changing (CC) weak current, and full $\mathrm{CC}$ current, are displayed for ${ }^{4} \mathrm{He}$ as function of the $v_{\mu}$ energy in the allowed kinematical range. The full $\mathrm{CC} 1 \mathrm{~b}$ and $2 \mathrm{~b}$ results for ${ }^{3} \mathrm{H}$ are compared to the $\mathrm{CC} 1 \mathrm{~b}$ results of Ref. [8] in the inset. The theoretical uncertainites resulting from combining statistical errors in the GFMC calculation with errors associated with the maximumentropy inversion of the imaginary-time data are shown by the bands. The arrow indicates the kinematically maximum allowed $E_{v}$, see text for further explanations.

Their implementation is also summarized in the Supplemental Material [59].

Predictions for the total rate in ${ }^{4} \mathrm{He}$ are compared to the experimental values and older theoretical estimates mentioned above in Table I, and the differential rates as functions of the energy of the muon neutrino emitted in the capture are shown for both ${ }^{3} \mathrm{H}$ and ${ }^{4} \mathrm{He}$ in Fig. 1. For ${ }^{4} \mathrm{He}$, results obtained by considering only the vector (V) or axial (A) components of the charge-changing $(\mathrm{CC})$ weak current and by including onebody (1b) terms only or both one- and two-body (2b) terms in these currents are listed in Table I, and displayed in Fig. 1, separately. Note that the response function $R_{x y}\left(E_{v}\right)$ in Eq. (9) involves interference between the matrix elements of the $\mathrm{V}$ and $\mathrm{A}$ currents, and therefore only contributes when both are present. As a consequence, $\Gamma(\mathrm{CC}) \neq \Gamma(\mathrm{V})+\Gamma(\mathrm{A})$; indeed, this $\mathrm{V}$-A interference leads to an increase in the $\Gamma(\mathrm{V})+\Gamma(\mathrm{A})$ result by $\approx 10 \%$ in both the $1 \mathrm{~b}$ - and $2 \mathrm{~b}$-based calculations. For ${ }^{3} \mathrm{H}$, only the full $\mathrm{CC} 1 \mathrm{~b}$ and $2 \mathrm{~b}$ GFMC, and full $\mathrm{CC} 1 \mathrm{~b}$ Faddeev [8] differential rates are displayed in the inset of Fig. 1. While their shapes differ somewhat in the threshold region (see below), the corresponding integrated rates are in excellent agreement with each other: the GFMC $1 \mathrm{~b}$ result is $32.4(6) \mathrm{s}^{-1}$, to be compared to the $32.6 \mathrm{~s}^{-1}$ reported in Ref. [8]. Two-body currents increase the total rate to 35.1(9) $\mathrm{s}^{-1}$. We focus on ${ }^{4} \mathrm{He}$ hereafter.

In the ${ }^{4} \mathrm{He}$ capture, the neutrino energy is in the range $0 \leqslant E_{v} \leqslant E_{v}^{\max } \approx 83.6 \mathrm{MeV}$; however, the distribution, on account of the $E_{v}^{2}$-weighing factor present in the expression for $d \Gamma / d E_{v}$, is skewed toward the high end, confirming the expectation that the energy release in the capture process is converted primarily into energy for the emitted neutrino [52] with the remaining balance being absorbed by the final nuclear system. In the present case, since ${ }^{4} \mathrm{H}$ is not bound, the possible final breakup channels are ${ }^{3} \mathrm{H}+n(3+1),{ }^{2} \mathrm{H}+2 n$ $(2+2)$, and ${ }^{1} \mathrm{H}+3 n(1+3)$, which have slightly different thresholds. While the contributions of these channels are fully accounted for here, they cannot be individually identified over the allowed $E_{v}$ range-a limitation intrinsic to the present method and apparent from Eq. (11), which relies on closure to remove the sum over final states. Despite relying on the closure approximation, Caine and Jones [56] estimated the branching ratios into the $3+1,2+2$, and $1+3$ channels to be, respectively, $97.75 \%, 2 \%$, and $0.25 \%$.

A related issue has to do with the behavior of the response functions in the threshold region $E_{v} \lesssim E_{v}^{\max }$. The kinematical constraint that $R_{\alpha \beta}\left(E_{v}\right)$ vanish for $E_{v}$ larger than $E_{v}^{\max }$ is not imposed (for both ${ }^{3} \mathrm{H}$ and ${ }^{4} \mathrm{He}$ ) when performing the inversion (see supplemental material). Even though relatively high values of $\tau \leqslant \tau^{\max }=0.1 \mathrm{MeV}^{-1}$ are calculated by GFMC, the maximum-entropy procedure we utilize still produces some strength beyond $E_{v}^{\max }$, as is apparent from Fig. 1. However, the integrals of $d \Gamma / d E_{v}$, when evaluated over the whole $E_{v}$ range including the unphysical region, remain stable to within $1 \%$ for $\tau^{\max }=(0.1,0.08,0.05) \mathrm{MeV}^{-1}$.

In Table I we also list the results for the $1 \mathrm{~b}$ and $2 \mathrm{~b}$ total inclusive rates (indicated as $\widetilde{C C}$ ) obtained with an incomplete $\mathrm{CC}$ weak current in which the term proportional to the induced pseudoscalar form factor $G_{P}\left(q^{2}\right)$ (in the axial sector) is ignored. The effect is significant: Retaining this term reduces the $\widetilde{C C}$ values by $\approx 15 \%(14 \%)$ in the $1 \mathrm{~b}(2 \mathrm{~b})$ calculations. The parametrization for $G_{P}\left(q^{2}\right)$ adopted here [51] is consistent with the recent determination of this form factor by the MuCap collaboration [16]. It also leads, in an accurate $a b$ initio calculation based on essentially the same dynamical inputs adopted here [60], to a prediction for the ${ }^{3} \mathrm{He}\left(\mu^{-}, v_{\mu}\right)^{3} \mathrm{H}$ total rate that is agreement with the (remarkably precise) measurement of Ref. [61], 1496(4) $\mathrm{s}^{-1}$. Thus, muon capture provides a sensitive test of the $G_{P}\left(q^{2}\right)$ form factor at low momentum transfers. By contrast, this observable is only very marginally affected (at a fraction of a $1 \%$ level) by changes in the parametrization of the nucleon axial form factor, as we have explicitly verified by calculating how the total rate changes when the cutoff $\Lambda_{A}$ is varied by $\pm 10 \%$ about its central value of $\Lambda_{A} \approx 1 \mathrm{GeV}$. The reason is that $G_{A}\left(q^{2}\right)=g_{A}\left[1+2 q^{2} / \Lambda_{A}^{2}+\cdots\right]$, and $q^{2} / \Lambda_{A}^{2} \ll 1$ in the allowed kinematical region.

In this work, we have formulated an $a b$ initio GFMC method for calculating inclusive muon-capture rates on light nuclei (mass number $A \leqslant 12$ ), and have presented, as first applications, calculations of the total rate in ${ }^{3} \mathrm{H}$ and ${ }^{4} \mathrm{He}$. The predicted value in ${ }^{4} \mathrm{He}$ is consistent with the lower range of available experimental determinations (see Table I). However, these measurements from bubble chamber experiments of the late 1960s have large errors, making it impossible to establish, at a quantitative level, the validity of the model for the nuclear charge-changing weak current we have adopted here. We hope the present work will motivate our colleagues to carry out a new experiment on ${ }^{4} \mathrm{He}$.

Future plans in this area include (i) the application of the method to other (light) nuclei, especially in cases where more accurate data are known [52], and (ii) its extension to more fundamental dynamical approaches based on interactions and 
electroweak currents derived from chiral effective field theory. The presence of discrete states in the final nuclear system substantially complicates the calculation of the capture rate, since the imaginary-time response functions $E_{\alpha \beta}(q, \tau)$ would have to be evaluated at large enough values of $\tau$ to reliably resolve the contributions of these states in the threshold region of the corresponding "inverted" $R_{\alpha \beta}\left(E_{v}\right)$-that is, when $E_{v}$ is close to the maximum kinematically allowed value. A similar issue arose in the calculation of the longitudinal and transverse electromagnetic response functions of ${ }^{12} \mathrm{C}$ [44].

\section{ACKNOWLEDGMENTS}

We acknowledge the support of the U.S. Department of Energy, Office of Science, Office of Nuclear Physics, under
Contracts No. DE-AC02-06CH11357 (A.L. and N.R.) and No. DE-AC05-06OR23177 (R.S.), as well as the support by the NUclear Computational Low-Energy Initiative (NUCLEI) SciDAC project (N.R.) and by the Fermi Research Alliance, LLC, under Contract No. DE-AC02-07CH11359 with the U.S. Department of Energy, Office of Science, Office of High Energy Physics (N.R.). Under an award of computer time provided by the INCITE program, this research used resources of the Argonne Leadership Computing Facility at Argonne National Laboratory, which is supported by the Office of Science of the U.S. Department of Energy under Contract No. DE-AC02-06CH11357. Computational resources provided by the National Energy Research Scientific Computing Center (NERSC) are also gratefully acknowledged.
[1] A. Czarnecki, G. P. Lepage, and W. J. Marciano, Phys. Rev. D 61, 073001 (2000).

[2] H. Primakoff, Rev. Mod. Phys. 31, 802 (1959).

[3] J. G. Congleton and H. W. Fearing, Nucl. Phys. A 552, 534 (1992).

[4] J. G. Congleton and E. Truhlik, Phys. Rev. C 53, 956 (1996).

[5] L. E. Marcucci, R. Schiavilla, S. Rosati, A. Kievsky, and M. Viviani, Phys. Rev. C 66, 054003 (2002).

[6] L. E. Marcucci, A. Kievsky, S. Rosati, R. Schiavilla, and M. Viviani, Phys. Rev. Lett. 108, 052502 (2012); 121, 049901(E) (2018).

[7] J. Golak, R. Skibinski, H. Witala, K. Topolnicki, A. E. Elmeshneb, H. Kamada, A. Nogga, and L. E. Marcucci, Phys. Rev. C 90, 024001 (2014).

[8] J. Golak, R. Skibinski, H. Witala, K. Topolnicki, H. Kamada, A. Nogga, and L. E. Marcucci, Phys. Rev. C 94, 034002 (2016).

[9] C. E. Hyde-Wright and K. de Jager, Annu. Rev. Nucl. Part. Sci. 54, 217 (2004).

[10] C. Patrignani et al. (Particle Data Group), Chin. Phys. C 40, 100001 (2016).

[11] E. Amaldi, S. Fubini, and G. Furlan, Electroproduction at Low Energy and Hadron Form Factors, Springer Tracts in Modern Physics, Vol. 83 (Springer-Verlag, Berlin, 1979), p. 1.

[12] L. A. Ahrens et al., Phys. Rev. D 35, 785 (1987).

[13] N. J. Baker, A. M. Cnops, P. L. Connolly, S. A. Kahn, H. G. Kirk, M. J. Murtagh, R. B. Palmer, N. P. Samios, and M. Tanaka, Phys. Rev. D 23, 2499 (1981).

[14] K. L. Miller et al., Phys. Rev. D 26, 537 (1982).

[15] T. Kitagaki et al., Phys. Rev. D 28, 436 (1983).

[16] V. A. Andreev et al. (MuCap Collaboration), Phys. Rev. Lett. 110, 012504 (2013).

[17] V. Bernard, N. Kaiser, and Ulf-G. Meissner, Phys. Rev. D 50, 6899 (1994)

[18] V. Bernard, L. Elouadrhiri, and U.-G. Meissner, J. Phys. G 28, R1 (2002).

[19] N. Kaiser, Phys. Rev. C 67, 027002 (2003).

[20] I. S. Towner, Phys. Rep. 155, 263 (1987).

[21] D. O. Riska, Phys. Rep. 181, 207 (1989).

[22] T.-S. Park, D.-P. Min, and M. Rho, Phys. Rep. 233, 341 (1993).

[23] T.-S. Park, D.-P. Min, and M. Rho, Nucl. Phys. A 596, 515 (1996).
[24] S. Pastore, L. Girlanda, R. Schiavilla, M. Viviani, and R. B. Wiringa, Phys. Rev. C 80, 034004 (2009)

[25] S. Pastore, L. Girlanda, R. Schiavilla, and M. Viviani, Phys. Rev. C 84, 024001 (2011).

[26] S. Kölling, E. Epelbaum, H. Krebs, and U.-G. Meissner, Phys. Rev. C 80, 045502 (2009); 84, 054008 (2011).

[27] M. Piarulli, L. Girlanda, L. E. Marcucci, S. Pastore, R. Schiavilla, and M. Viviani, Phys. Rev. C 87, 014006 (2013).

[28] A. Baroni, L. Girlanda, S. Pastore, R. Schiavilla, and M. Viviani, Phys. Rev. C 93, 015501 (2016); 93, 049902(E) (2016); 95, 059901(E) (2017).

[29] H. Krebs, E. Epelbaum, and U.-G. Meissner, Ann. Phys. 378, 317 (2017).

[30] J. Carlson and R. Schiavilla, Rev. Mod. Phys. 70, 743 (1998).

[31] S. Bacca and S. Pastore, J. Phys. G: Nucl. Part. Phys. 41, 123002 (2014)

[32] J. Carlson, S. Gandolfi, F. Pederiva, S. C. Pieper, R. Schiavilla, K. E. Schmidt, and R. B. Wiringa, Rev. Mod. Phys. 87, 1067 (2015).

[33] R. Schiavilla et al., Phys. Rev. C 58, 1263 (1998).

[34] A. Baroni et al., Phys. Rev. C 98, 044003 (2018).

[35] S. Pastore, A. Baroni, J. Carlson, S. Gandolfi, S. C. Pieper, R. Schiavilla, and R. B. Wiringa, Phys. Rev. C 97, 022501(R) (2018).

[36] P. Gysbers et al., Nat. Phys. 15, 428 (2019).

[37] E. Kolbe, K. Langanke, and P. Vogel, Phys. Rev. C 50, 2576 (1994).

[38] N. T. Zinner, K. Langanke, and P. Vogel, Phys. Rev. C 74, 024326 (2006).

[39] T. Marketin, N. Paar, T. Niksic, and D. Vretenar, Phys. Rev. C 79, 054323 (2009).

[40] J. C. Hardy and I. S. Towner, Phys. Rev. C 91, 025501 (2015).

[41] J. D. Walecka, Theoretical Nuclear and Subnuclear Physics, 2nd ed. (Imperial College Press and World Scientific Publishing, London, 2002), p. 489.

[42] A. Lovato, S. Gandolfi, R. Butler, J. Carlson, E. Lusk, S. C. Pieper, and R. Schiavilla, Phys. Rev. Lett. 111, 092501 (2013).

[43] A. Lovato, S. Gandolfi, J. Carlson, S. C. Pieper, and R. Schiavilla, Phys. Rev. C 91, 062501(R) (2015). 
[44] A. Lovato, S. Gandolfi, J. Carlson, S. C. Pieper, and R. Schiavilla, Phys. Rev. Lett. 117, 082501 (2016).

[45] A. Lovato, S. Gandolfi, J. Carlson, E. Lusk, S. C. Pieper, and R. Schiavilla, Phys. Rev. C 97, 022502(R) (2018).

[46] J. Carlson and R. Schiavilla, Phys. Rev. Lett. 68, 3682 (1992).

[47] M. Jarrell and J. E. Gubernatis, Phys. Rep. 269, 133 (1996).

[48] R. K. Bryan, Eur. Biophys. J. 18, 165 (1990).

[49] R. B. Wiringa, V. G. J. Stoks, and R. Schiavilla, Phys. Rev. C 51, 38 (1995).

[50] S. C. Pieper, AIP Conf. Proc. 1011, 143 (2008).

[51] G. Shen, L. E. Marcucci, J. Carlson, S. Gandolfi, and R. Schiavilla, Phys. Rev. C 86, 035503 (2012).

[52] D. F. Measday, Phys. Rep. 354, 243 (2001).

[53] R. Bizzari, E. di Capua, U. Dore, G. Gialanella, P. Guidoni, and I. Laakso, Nuovo Cimento 33, 1497 (1964).
[54] L. B. Auerbach, R. J. Esterling, R. E. Hill, D. A. Jenkins, J. T. Lach, and N. H. Lipman, Phys. Rev. 138, B127 (1965).

[55] M. M. Block, T. Kikuchi, D. Koetke, C. R. Sun, R. Walker, G. Gulligan, V. L. Telegdi, and R. Winston, Nuovo Cimento A (1971-1996) 55, 501 (1968).

[56] C. A. Caine and P. S. H. Jones, Nucl. Phys. 44, 177 (1963).

[57] J. D. Walecka, in Muon Physics III, edited by V. W. Hughes and C. S. Wu (Academic Press, New York, 1975).

[58] J. Carlson, Phys. Rev. C 36, 2026 (1987).

[59] See Supplemental Material at http://link.aps.org/supplemental/ 10.1103/PhysRevC.100.035502 for a more detailed account of GFMC calculations of the Euclidean response functions and of the maximum-entropy technique.

[60] L. E. Marcucci, M. Piarulli, M. Viviani, L. Girlanda, A. Kievsky, S. Rosati, and R. Schiavilla, Phys. Rev. C 83, 014002 (2011).

[61] P. Ackerbauer et al., Phys. Lett. B 417, 224 (1998). 